\title{
Non-Invasive Stimulation-Based Tactile Sensation for Upper-Extremity Prosthesis: A Review
}

\author{
Kairu Li, Student Member, IEEE, Yinfeng Fang, Member, IEEE, Yu Zhou, Student Member, IEEE, \\ and Honghai Liu, Senior Member, IEEE
}

\begin{abstract}
An ideal hand prosthesis should provide satisfying functionality based on reliable decoding of the user's intentions and deliver tactile feedback in a natural manner. The absence of tactile feedback impedes the functionality and efficiency of dexterous hand prostheses, which leads to a high rejection rate from prostheses users. Thus, it is expected that integration of tactile feedback with hand prostheses will improve the manipulation performance and enhance perceptual embodiment for users. This paper reviews the state-of-the-art of non-invasive stimulationbased tactile sensation for upper-extremity prostheses, from the physiology of the human skin, to tactile sensing techniques, noninvasive tactile stimulation, and an emphasis on electrotactile feedback. The paper concludes with a detailed discussion of recent applications, challenging issues, and future developments.
\end{abstract}

Index Terms-Prosthetic hand, tactile transduction techniques, non-invasive stimulation feedback, electrotactile stimulation.

\section{INTRODUCTION}

A $\mathrm{N}$ upper-extremity prosthesis can be a substitute to restore the body appearance and hand capability for one with amputation or congenital limb deficiency. Regarding hand capability, an ideal prosthetic hand should deliver functionality of grasp or manipulation, and have tactile sensation to explore surrounding objects in human-centered environments. However, few hand prostheses can provide efficient tactile feedback to users. The absence of tactile sensation leads to unreliable prosthetic manipulation performance and a high rejection rate of prosthetic hands from customers [1]-[3], which substantially constrains hand prostheses from being commercially viable.

Presently, most hand prostheses focus on improving the mechanical structure or prosthetic control strategies for better manipulation performance, while neither commercial nor lab prosthetic hands provide satisfactory tactile sensation to users. Taking dominant commercial hand prostheses for example, such as i-Limb by Touch Bionics [4], Myohand and Michelangelo hand by Ottobock [5], and the Bebionic hand by steeper [6], [7], their multiple degrees of freedom (DOFs) enable users to accomplish fundamental tasks in daily life,

Manuscript received January 6, 2017; revised February 15, 2017; accepted February 15, 2017. This work was supported by the China Scholarship Council under Grant. 201508060299. The associate editor coordinating the review of this paper and approving it for publication was Prof. Subhas Chandra Mukhopadhyay. (Corresponding author: Honghai Liu.)

$\mathrm{K}$. Li, Y. Fang, and H. Liu are with the School of Computing, University of Portsmouth, Portsmouth, PO1 3HE, U.K. (e-mail: kairu.li@port.ac.uk; yinfeng.fang@port.ac.uk; honghai.liu@port.ac.uk).

Y. Zhou is with the School of Mechanical Engineering, Shanghai Jiao Tong University, Shanghai 200240, China (e-mail: hnllyu@sjtu.edu.cn).

Digital Object Identifier 10.1109/JSEN.2017.2674965 however, they are unable to make users aware of the tactile information without visual feedback. VINCENTevolution 2 might be the only one commercialized hand prosthesis with a stimulation system for force feedback [8], but no customer reviews are found to confirm the effectiveness of its feedback performance for now. Available lab artificial hands are integrated with various sensors. For example, force/pressure sensors and torque sensors are utilized in DLR II hand [9], Southampton Hand [10], [11], LUKE hand [12] and Cyerhand [13] for slip prevention and finger position feedback. Southampton hand and Shadow hand [14] are also integrated with temperature sensors. Besides, micro-vibration sensors are optional on Shadow hand. However, the tactile information acquired by sensors is only fed back to prostheses themselves to pursue a stable control performance, instead of providing tactile feedback to users. Encouragingly, an invasive tactile system was recently tested on self-controlled prostheses users to provide limited tactile feedback [15], [16]. Thus, there still remains great challenges of a full restoration of tactile sensation for hand prostheses, although preliminary success has been achieved.

Prosthetic hands benefit from tactile sensation in many aspects. Apart from increasing the prosthesis acceptance rate, the integration is suggested to prevent slip [17], [18] and significantly increase the success rate of applying correct grasping forces [19]. Also, tactile stimulation could help to alleviate phantom pain, muscle fatigue, and generate a sense of body ownership for prosthesis users [1], [20]. Thus, tactile sensation restoration for hand prostheses is helpful to improve user experience and manipulation performance.

The restoration of tactile sensation for hand prostheses can be outlined into two steps: tactile sensing and stimulation feedback. Tactile sensing based on different transduction techniques aims to detect and measure a given property of an object through contact [21]. This is usually conducted by tactile sensors attached on fingertips or palms of prosthetic hands, such as force sensors or artificial skin covering the whole hand. With regard to tactile feedback, it is to send tactile information to a user's residual body for perceptual interpretation and is usually conducted by stimulation techniques. Depending on whether stimulation electrodes are implanted into the skin, tactile stimulation can be classified as invasive (e.g. direct neural stimulation) and non-invasive (surface stimulation). In theory, direct neural stimulation with neural electrodes implanted in the peripheral nervous system (PNS) may potentially generate natural tactile feelings. The 20-channel invasive system mentioned above could generate 
TABLE I

Properties of Four Types of Mechanoreceptors in Human Skin (Summarized From [17], [25], [30], And [31])

\begin{tabular}{lllll}
\hline & Meissner corpuscles & Pacinian corpuscles & Merkel cells & Ruffini endings \\
\hline Classification & FA I & FA II & SA I & SA II \\
Adaptation rate & Fast & Fast & Slow & Slow \\
Receptive field & Small and sharp & Large and diffuse & Small and sharp & Large and diffuse \\
Density (units $\left./ \mathrm{cm}^{2}\right)$ & 140 & 20 & 70 & 0.5 \\
Spatial resolution $(\mathrm{mm})$ & $3-4$ & $10+$ & $0.4-40$ & $7+$ \\
Stimuli frequency $(\mathrm{Hz})$ & $5-50$ & $40-500+$ & Low frequency & $<7$ \\
Sensory function & High frequency & High frequency & Low frequency \\
& vibration detection. & vibration detection; & vibration detection (static force); vibration detection (static force); \\
& Temporal changes in & Temporal changes in & Pattern/form detection; & Finger position; \\
& skin deformation. & skin deformation. & Texture perception; & Stable grasp; \\
& & Tool use. & Tactile flow perception. & Tangential force/ skin stretch. \\
\hline
\end{tabular}

feelings of pulsing pressure, constant pressure, tapping, 2 types of texture, and objects moving at 19 small places on a subject's hand, such as their palm, wrist, and fingertips [16]. Further studies are expected to restore natural feeling over the whole hand. However, invasive stimulation suffers from risks of infection and rejection, poor knowledge of neural decoding, technical issues of surgery and electrode replacement, and so on. Given above scenarios, this study gives priority to noninvasive stimulation feedback, mainly including electrotactile stimulation, vibrotactile stimulation, and mechanotactile stimulation. For direct neural stimulation, please refer to [15], [16], [22], [23]. In recent decades, studies about tactile sensation restoration is boosted by advancements in computation and sensor fabrication techniques, however, these studies are not well implemented in current hand prostheses on account of technical difficulties and the complicated nature of human tactile sensation.

Despite existing literature of tactile sensing on robotics [17], [18], [24], biomedical engineering [25], [26], and tactile stimulation on prosthetic hands [27], [28], it is required to have a comprehensive understanding for the whole process of tactile sensation restoration consisting of sensing, stimulating, and related applications to pave the way for further study, especially in the context of upper-extremity prosthetic devices. This paper aims at filling this gap and is organized as follows. Section II introduces the physiology of the human tactile sensing system and generalized design requirements for artificial sensors. The state-of-the-art techniques of tactile sensing and non-invasive tactile stimulation are respectively reviewed in Sections III and IV. Considering the big potential of electrotactile stimulation, electrotactile feedback driven studies, and its applications in the context of the upper extremity prostheses are further presented in Section V. The paper concludes with a discussion of challenges and future directions in Section VI.

\section{Physiology OF Human Skin}

The human skin is capable of sensing touch, consisting of mechanical stimulation, heat, and pain. This section primarily focuses on mechanical stimulation, corresponding to tactile sensations of force, shape, texture, stiffness, etc. Mechanoreceptors are sensory units distributed in human skin to detect mechanical stimulations such as force, pressure and vibration. When a mechanoreceptor detects an external

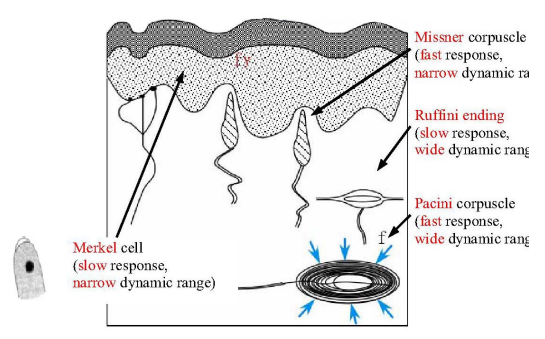

Fig. 1. Four types of mechanoreceptors in human hands [29] and their receptive fields [30].

stimulation, a sequence of voltage pulses is generated and transmitted through neurons to the brain where the information is processed.

Mechanoreceptors in the glabrous skin of human hands include four types: Merkel cells, Meissner corpuscles, Ruffini endings, and Pacinian corpuscles. They are responsible for the detection of different stimulations. Generally, according to their adaptation rate, four types of mechanoreceptors are categorized into two classes: fast adapting units (FA) and slow adapting units (SA). Then, based on their receptive fields, each class is divided into two groups: I and II. SA I and FA I receptors have small receptive field with a sharp border, while SA II and FA II receptors have large receptive field with diffuse border, as shown in Fig. 1 [29], [30].

These four types of mechanoreceptors have different functional properties with regards to the receptive speed, the receptive field, and the perceptive function, which are summarized in Table I. In terms of the receptive speed, Meissner corpuscles and Pacinian corpuscles are mainly responsible for rapid or dynamic stimulation, while Merkel cells and Ruffini endings respond to sustained stimulation. Meissner corpuscles are sensitive to light touch, while Pacinian corpuscles tend to detect deep pressure touch and high frequency vibration. Merkel cells are sensitive to low frequency vibration, while Ruffini endings usually respond to streching of the skin. In terms of the location and the receptive field, Meissner corpuscles and Merkel cells concentrate in the outer layer of the skin on fingertips and have small receptive fields. On the contrary, Pacinian corpuscles and Ruffini endings are distributed more uniformly in deep layer of the skin on fingers and the palm. In terms of the function of perception, Merkel cells and Pacinian corpuscles might be related to 
TABLE II

Characteristics of Tactile Sensors Based on Different Transduction Techniques

\begin{tabular}{|c|c|c|c|}
\hline Tactile sensors & Working principle & Advantage & Disadvantage \\
\hline Strain gauges & $\begin{array}{l}\text { Its resistance varies with the ap- } \\
\text { plied stress. }\end{array}$ & $\begin{array}{l}\text { High spatial resolution; High strain sensitivity; } \\
\text { Widely used, well developed; Low cost. }\end{array}$ & $\begin{array}{l}\text { Non-linear; Sensitive to temperature and hu- } \\
\text { midity; Inability to sense stable value. }\end{array}$ \\
\hline Piezoresistors & $\begin{array}{l}\text { Its resistance varies with the defor- } \\
\text { mation caused by applied force. }\end{array}$ & $\begin{array}{l}\text { Simple electronics; High sensitivity; Ease of in- } \\
\text { tegrating in MEMS; Resistant to interference. }\end{array}$ & $\begin{array}{l}\text { Hysteresis; Temperature sensitivity; Fragile } \\
\text { and rigid; High cost. }\end{array}$ \\
\hline $\begin{array}{l}\text { Capacitive } \\
\text { sensors }\end{array}$ & $\begin{array}{l}\text { Its capacitance varies with the de- } \\
\text { formation caused by applied force. }\end{array}$ & $\begin{array}{l}\text { Sensitivity of small force change; Reliability; } \\
\text { Large dynamic range, suitable for both dynamic } \\
\text { and static force measurement; Low temperature } \\
\text { sensitivity; Low power consumption. }\end{array}$ & $\begin{array}{l}\text { Limited spatial resolution; Noise sensitivity; } \\
\text { Complex electronics. }\end{array}$ \\
\hline $\begin{array}{l}\text { Piezoelectric } \\
\text { sensors }\end{array}$ & $\begin{array}{l}\text { An electric voltage will be pro- } \\
\text { duced when a force applied to it. }\end{array}$ & $\begin{array}{l}\text { No need for power supply; High reliability; Fast } \\
\text { dynamic response, able to measure vibration. }\end{array}$ & $\begin{array}{l}\text { Low spatial resolution; High temperature sen- } \\
\text { sitivity; Inability to sense static value. }\end{array}$ \\
\hline $\begin{array}{l}\text { Optical } \\
\text { sensors }\end{array}$ & $\begin{array}{l}\text { The intensity or the spectrum of } \\
\text { light varies with the applied force. }\end{array}$ & $\begin{array}{l}\text { Immune to electromagnetic field; High spatial } \\
\text { resolution. }\end{array}$ & $\begin{array}{l}\text { Fragile and rigid; Large size; Inability to trans- } \\
\text { parency and highly reflective surface. }\end{array}$ \\
\hline
\end{tabular}

the sensation of stiffness. Merkel cells and Ruffini endings could detect slip and shape due to their response to steady pressure and skin stretch. Besides, Meissner corpuscles and Pacinian corpuscles contribute to the perception of texture, such as surface roughness, because they are sensitive to rapid vibration which is too small to activate the other two types of mechanoreceptors [30]. Additionally, the spatial resolution is the smallest distance for one to distinguish two-point touch and varies across the body. It is as close as $0.5 \mathrm{~mm}$ on fingertips while $7 \mathrm{~mm}$ on the palm.

The human skin can be an ideal model of artificial tactile sensors given its good performance of tactile sensing. In general, artificial sensors are expected to demonstrate small resolution, high sensitivity, low hysteresis, fast and linear response, wide dynamic range and high reliability. A spatial resolution of $5-40 \mathrm{~mm}$ could be satisfactory. Typically, $20-60 \mathrm{~Hz}$ would be fine for sampling rate in common tasks, while for special task, such as texture recognition, a higher sampling rate about $1-2.5 \mathrm{kHz}$ is necessary [17]. A force sensitivity range of $0.3-10 \mathrm{~N}$ is required. For human-like skin or sensors, robust, flexible, stretchable and soft materials are desired to be embedded on various 3D structures. Additionally, low cost, low power consumption and scalability are also important for manufacture and implementation.

\section{TACTILE SENSING}

Tactile sensing is the first step of the restoration of tactile sensation. A increasing demand for tactile sensation feedback in recent decades inspires the exploration of transduction techniques [26], [32], [33] and their applications in various systems, such as upper limb prostheses [34], [35], virtual reality systems [36], [37], remote operation in dangerous environments [38], minimally invasive surgery (MIS) [39], nanometerscale operations [40], [41], surgical training [42], touch screens [43], and robotic hands [24], [44], [45]. The study of tactile sensing in upper limb prostheses is not as mature as that in other fields, but their achievements could be adopted into this field. Given that grasping is one of the major functions of hands, most studies of prosthesis tactile sensing focus on grasp force or pressure in order to prevent slip and achieve a stable grasp. The measured characteristics of touch, however, can be not only force and pressure, but also stiffness, texture, or shape. Thus, different transduction techniques are desired to be synthesized to realize a humanlike tactile sensing system. This section presents available tactile sensing techniques which have potential to be applied in hand prostheses, namely, resistive sensors (strain gauges and piezoresistors), capacitive sensors, piezoelectric sensors, optical sensors and artificial skins. Characteristics of various tactile sensors are summarized in Table II and they are further discussed in the following subsections.

\section{A. Resistive Sensors}

1) Strain Gauges: A strain gauge is a device adhered on the surface of an object to measure the strain caused by external pressure. Most of strain gauges are based on a resistive foil pattern which is mounted on a backing material, and both of the foil and the backing material are attached by different glues depending on required lifetime. The resistance of the foil changes with the stress applied on it.

Strain gauges are more suitable to measure dynamic strains rather than static ones [46], because of high temperature and humidity sensitivities. Wheatstone bridge configurations are usually introduced to compensate environmental changes [26]. Strain gauges also exhibit nonlinear response. Generally, the smaller a strain gauge is, the higher accuracy can be achieved, because the measured strain is the average strain over the gauge length. Besides, sensors of smaller size are flexible and robust to be applied over dexterous surfaces, such as prostheses, robots and medical devices [47]. Micromachined strain gauges based on metal and semiconductor have been realized with the development of manufacturing technologies, although it is not easy to fabricate and handle tiny gauges [46], [48]. Metal-based and semiconductor-based strain gauges show many advantages, such as high spatial resolution and high strain sensitivity. For example, a nanofiberbased strain gauge, which is able to detect pressure, shear and torsion, was proposed to be flexible and sensitive even to human heartbeats [49]. Strain gauges have been popularly used in various sensors, such as pressure sensors, torque sensors and 


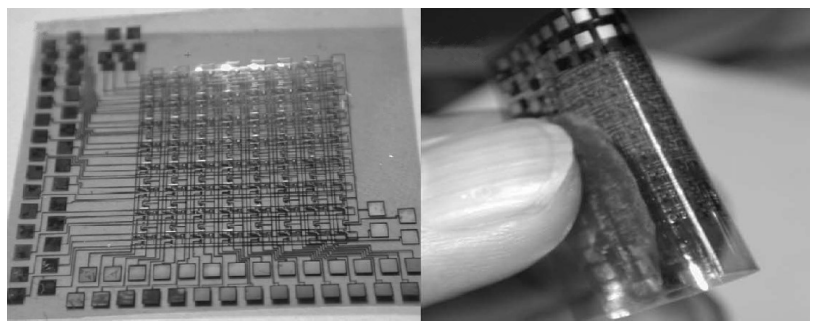

Fig. 2. Flexible tactile sensor based on strain gauges [50].

position sensors, which indicates a major advantage of strain gauges in terms of their well-established fabrication techniques and applications.

Da Silva et al. proposed a finger-mounted tactile sensor based on the strain gauge which presented a linear response, a wide force sensitivity of $0-100 \mathrm{~N}$ with a resolution of $0.3 \mathrm{~N}$, and a low hysteresis of $1.7 \%$ [48]. As shown in Fig. 2, another flexible strain gauge sensor was fabricated for the detection of normal and shear force, which could be measured by the voltage drop in strain gauges [50]. This sensor had a simple structure, but it was less sensitive to small forces.

2) Piezoresistors: Piezoresistive tactile sensors also belongs to resistive sensors. Its resistance varies with the deformation caused by the applied force on it, so the force can be obtained by the measurement in a piezoresistor's resistance. Generally speaking, piezoresistors are made of metals, semiconductors, and mainly silicon. Due to the easy measurement of resistance, piezoresistive tactile sensors have friendly electronic interface. They exhibit good sensitivity and are less susceptible to interference [26]. Another advantage is the easiness to be implemented in microelectromechanical systems (MEMSs) or integrated to printed circuit boards [51]. Despite the mentioned advantages, piezoresistors suffer from hysteresis, temperature sensitivity, fragility, rigidity and high cost [51]. Some efforts have been made to overcome the problem of fracture by embedding piezoresistors in flexible thin films [52] or polymers [53], [54].

Jorgovanovic et al. [113] presented the static and dynamic characterization of piezoresistive sensors used for detecting the positions of prosthetic finger joints. The feasibility of wireless communication between sensors and a receiving device, to reduce wires, was also discussed. Kane et al. [56] proposed a piezoresistive stress sensor array with high spatial resolution comparable to human dermis $(\approx 300 \mu \mathrm{m})$. It exhibited high potential for dexterous manipulation applications. Various applications with piezoresistive tactile sensors can also be found in stress and force measurement [57], [58], stiffness of soft tissues detection [59], fingertip sensing [60], etc.

\section{B. Capacitive Sensors}

A capacitive sensor is among the most sensitive sensors for detecting small force changes. It generally consists of two parallel conductive layers which are separated by dielectric materials. When force is applied on the capacitors, the capacitance between the layers varies with the reduced distance between layers and the deformation of the middle dielectric material as well [61]. Capacitive sensors exhibit high sensitivity, robust performance, a large dynamic range [62], lower

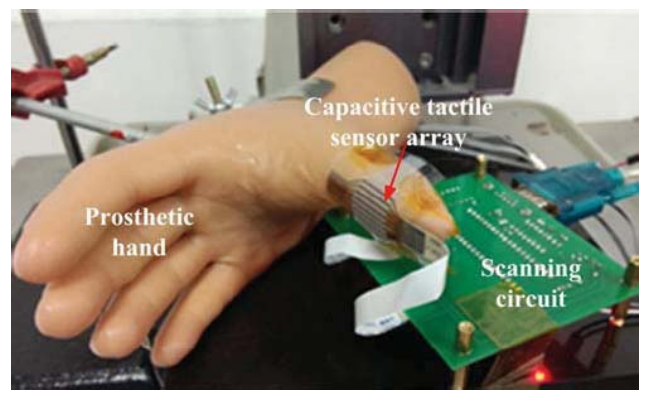

Fig. 3. Capacitive tactile sensor array integrated into a prosthetic hand thumb finger [67].

temperature sensitivity and low power consumption [63]. It can be used for both dynamic and static force measurement [64]. In order to accurately measure the change in capacitance, the size of capacitors should not be too small, which, however, may limit their spatial resolution [62]. Additionally, their sensitivity to noise leads to relatively complex electronics for noise filtration. Capacitive sensors are considered as effective sensing elements and have been applied to multi-axis force measurement for gripping and objects manipulation [65], texture recognition [63] and touch screen application [43], etc.

A capacitive sensor for shear sensing was proposed with a size of $4 \mathrm{~N}$ [66]. It showed a high repeatability and approximately linear output within $\pm 2 \mathrm{~N}$, however, its dimension (3.5 $\mathrm{mm} \times 1.6 \mathrm{~mm} \times 1.6 \mathrm{~mm}$ ) was a point to be considered in practical applications. Another capacitive tactile sensor was presented for gripping force measurement with a sensor range of $0-3000 \mathrm{mN}$ [67]. It was tested on a prosthetic hand as shown in Fig. 3.

\section{Piezoelectric Sensors}

Piezoelectric effect is the ability of certain materials to generate an electrical charge in response to external mechanical stress. A piezoelectric tactile sensor is a device based on piezoelectric effect to measure changes, such as force, by converting them to an electrical voltage. Measurement in voltage mode is the simplest way to obtain the applied force. Besides, current measurement and shock wave measurement can be utilized as well [68].

Piezoelectric sensing is one of the few sensing techniques that do not require power supply, which is considered as an outstanding advantage. Besides, it also exhibits high sensitivity, reliability and fast dynamic response. A wide response range of 0 to $1 \mathrm{kHz}$ enables it to be a good choice for vibrations measurement [69]. However, due to the decrease of the output voltage, piezoelectric sensors are unsuitable for measuring static force and show low spatial resolution and poor temperature stability [62], [69].

Various piezoelectric materials can be used for constructing piezoelectric tactile sensors. One of the most widely used one is polyvinylidene fluoride (PVDF). PVDF is a semicrystalline polymer consisting of long chain molecules with repeated unit CF-CH. Its strong piezoelectricity is attributed to the high electronegativity of fluoride atoms comparing with carbon atoms which leads to a large dipole moment [70]. PVDF has many advantages [71], [72]: mechanical flexibility, 
dimensional stability, high piezoelectric coefficients, low weight, formability into very thin sheets $(5 \mu \mathrm{m})$ and relatively low price. Another promising piezoelectric material is zinc oxide $(\mathrm{ZnO})$ nanotransducer because of its high flexibility and bio-compatibility [73]. Also, its ability to generate electrical power when subjected to mechanical vibration leads to various potential applications, including wearable and self-power medical devices [74]. $\mathrm{ZnO}$ is proposed to be a good candidate material for pressure and temperature sensor to be applied to prosthetic limbs [75]. During the past years, piezoelectric sensors have been used in prosthetic hands for the detection of slip [76], texture [77] and stiffness [78].

\section{Optical Sensors}

An optical fiber force sensor generally consists of a light source, a transduction medium, and an optical detector, which is often a vision sensor or a photodiode. The light generated by the light source, usually light emitting diodes (LEDs), passes through the transduction medium which includes optical fibers and a modulator, and finally reaches the detector [79], [80]. Then the detector circuit converts the light signal into electrical signal to be further processed by following electronic devices. The intensity or the spectrum of the modulated light changes according to the variation of the applied force, which is the working principle of optical sensors.

Many electronics-based sensing techniques cannot be applied in magnetic environments because of the electromagnetic interference, however, optical sensing is one of few techniques that are immune to electromagnetic field [81]. This major advantage enables optical sensors to be used in minimally invasive surgeries (MISs) where magnetic resonant imaging (MRI) are widely used to provide high quality images of living organs [79], [82]. In addition, optical sensors have simple and compact structure, and high spatial resolution [83].

Despite of aforementioned attractive characteristics, there are several limitations in optical sensors. Most optical fibers are fragile and not as flexible as electric wires due to their rigidity. Also, their complexity and relatively large size is another problem to be considered for dexterous hand applications. Some solutions are proposed in [84] and [85]. For example, plastic optical fibers were used to overcome the rigidity problem and prevent the damage of optical sensors [86]. Efforts were also made to reduce the size of optical sensors by using only one LED matrices instead of two as both the light source and detector [87]. Additionally, optical sensors were applied in a scalable tactile sensor skin to cover the whole body of a robot, which demonstrated the sensors could be made with high flexibility and compliance [88]. In [89], a LED-based optical sensor prototype was mounted between fingers of a prosthetic hand as shown in Fig. 4. It was tested on surfaces with different properties which included roughness, curvature, and stiffness for slip detection. It failed to detect any motion for transparency surface (e.g. glass) and highly reflective surface (such as a front silvered mirror and CD), which might be overcome by a laser-based optical sensor.

\section{E. Artificial Skins}

In addition to various tactile sensors, efforts have also been made in studies of artificial skin through sensor fusion and

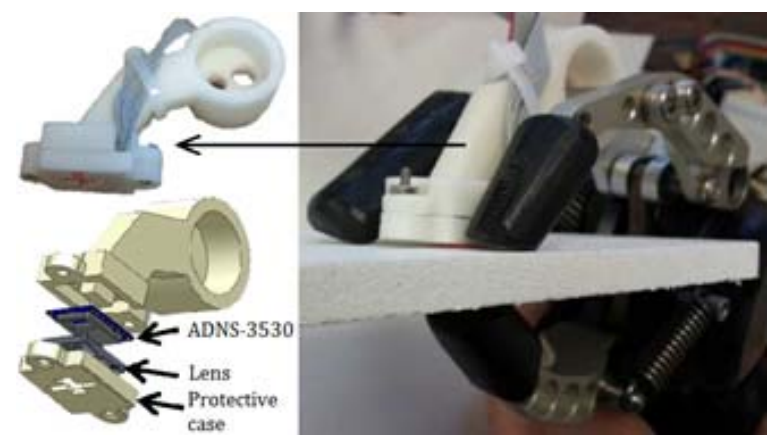

Fig. 4. Prototype of an optical sensor applied with a prosthetic hand [89].

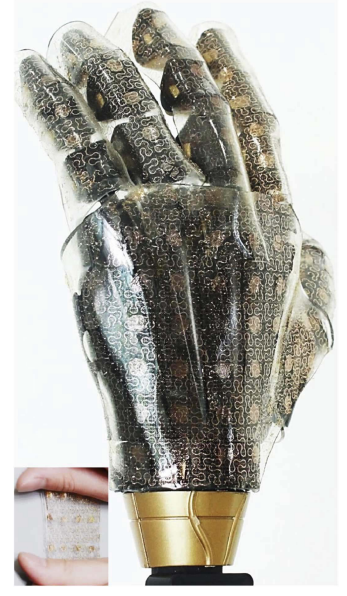

Fig. 5. Artificial skin [90].

the imitation of mechanoreceptors in the skin. A stretchable artificial skin, as shown in Fig. 5 [90], assembled pressure, temperature and humidity sensor arrays and was fabricated within ultrathin single crystalline silicon nanoribbons. It also integrated with electroresistive heaters which could be warmed to $36.5{ }^{\circ} \mathrm{C}$ to facilitate native skin perception. Furthermore, researchers connected the smart skin's sensors to a rat's peripheral nerves. The results showed that the sensory signal was successfully transferred to the rat's brain which indicated that this research could provide opportunities for PNS interfaces and enable amputees to feel various external stimulations. With regard to the imitation of mechanoreceptors in human skin, [29] proposed a four-layer arrayed capacitive sensor by reference to the four types of mechanoreceptors in human skin. Also, a power-efficient piezoresistive sensor was presented to mimic the SA mechanoreceptors for static pressure feedback [91]. The output of the sensors was used to stimulate somatosensory neurons through an optical/neural interface for pressure feedback. This study was considered to pave the way for the design and use of large-area organic electronic skins with tactile feedback for limb prostheses, although it was based on direct neural stimulation.

\section{Non-Invasive Tactile Stimulation}

Tactile stimulation is intended to make subjects aware of the tactile information detected by tactile sensors. This section introduces dominant non-invasive stimulation techniques, including electrotactile stimulation, vibrotactile stimulation, mechanotactile stimulation and contactless techniques applied for tactile feedback. 


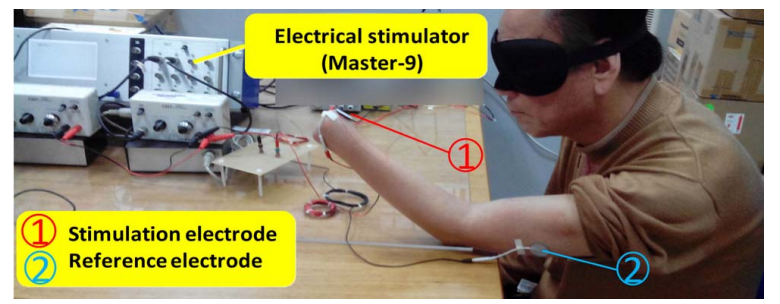

Fig. 6. Experimental setup for TENS: electrodes on the residual limb of the amputee [98].

\section{A. Electrotactile Stimulation}

There are various applications of electrical stimulation in the area of rehabilitation depending on the applied intensity. A more intensive stimulation is termed as functional electrical stimulation (FES), which has been used for motor recovery for stroke patients and prosthesis users [92], [93] by actuating muscle contraction. A slight one, which is not intensive enough to actuate muscles but can make users aware of, is utilized for tactile feedback and called electrotactile stimulation.

Electrotactile stimulation provides sensations by passing a local electric current to stimulate afferent nerve in the skin with surface electrodes. The modulated parameters include frequency, amplitude, pulse width and so on. Mulvey et al. proposed that transcutaneous electrical nerve stimulation (TENS) could generate a sensation on human skin by directing electrical pulses across the skin surface [94]. It is mostly used to reduce phantom pain and stump pain [95]. Initial experiments were conducted to support that TENS could be projected into a prosthetic hand and could enhance its sense of perceptual embodiment [96]. A recent study revealed that TENS could generate a strong sensation, although its effect on perceptual embodiment was modest [97]. It was tested to generate tactile sensation on the residual limbs of 11 amputees with their eyes covered as shown in Fig. 6 [98]. However, most research is still based on able-bodied participants and vision feedback is still a major factor for perceptual embodiment. More clinical tests are expected for evaluation in further experiments.

Electrotactile feedback is a direct way to stimulate PNS and is potential to generate natural tactile perception. Due to no mechanical parts, electrotactile stimulation has advantages of lower power consumption, light weight, and little noise compared with other tactile feedback techniques [27]. Despite many advantages, some unexpected feelings such as burning pain may result from electrotactile stimulation, which can be ameliorated by voltage-regulated stimulation and large electrodes. Additionally, another major drawback of electrotactile stimulation is its interference with electromyography (EMG) signal and electroencephalography (EEG) signal, although there are cases which tested electrotactile stimulation with EMG-based and EEG-based rehabilitation system [99], [100]. Studies about how to eliminate the interference when applied with myoelectric prostheses and EEG-based prostheses are discussed in section V-B.

\section{B. Vibrotactile Stimulation}

Vibrotactile stimulation is generated by mechanical vibration normal or transverse on the skin surface to convey tactile

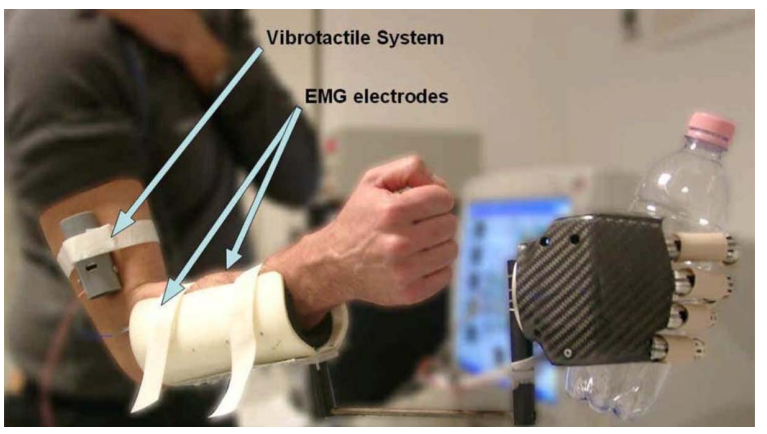

Fig. 7. Vibrotactile stimulation system applied with a myoelectric prosthetic hand [108].

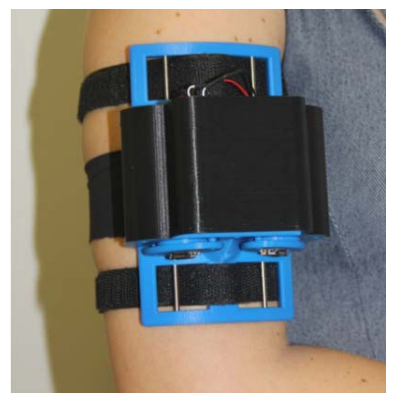

Fig. 8. Mechanotactile stimulator [109].

information through modulating vibration frequency, amplitude, duration, etc. [101]. It was firstly applied with prosthetic hands in 1953 [27], [102] and is considered to be suitable for myoelectric prostheses [103] and EEG-based prostheses [104] because of no interference with electric signal. The sensitivity to vibrotactile stimulation varies with the age and physical condition of subjects, and stimulation positions and frequency. Generally, the frequency ranges from $50 \mathrm{~Hz}$ to $300 \mathrm{~Hz}$ [105]. Currently, vibrotactile stimulation are widely used in cell phones and devices to assist deaf or blind people [106], [107]. A vibrotactile stimulation system applied with a myoelectric prosthetic hand is illustrated in Fig. 7 [108].

\section{Mechanotactile Stimulation}

Mechanotactile stimulation is to provide users with a force/pressure or position feedback while the movement of their prostheses. Comparing with electrotactile stimulation and vibrotactile stimulation, mechanotactile stimulation is able to generate a natural feeling of force/pressure, but the generated stimulation is applied in a different area (the residual body of the subject) from the original stimulus. As shown in Fig. 8 [109], a wearable mechanotactile stimulator is demonstrated to provide pressure and skin stretch information to the subject's residual limb. Current mechanotactile devices still have relatively large size, weight and high energy consumption when compared to vibrotactile or electrotactile devices [28]. Thus, further minimization is desired for mechanotactile stimulation devices.

\section{Others}

Some contactless techniques were also utilized for tactile feedback, such as magnetic-field [110], air-jet, airborne ultrasound [111], and infrared [112]. These studies are still at 
lab stage and are not adopted as widely as aforementioned stimulations. Their bulky dimension might be a challenge in daily use for upper extremity prostheses, but, with the development of technologies, they are potential to be applied in rehabilitation, virtual reality and so on.

\section{Electrotactile Feedback for UPPER-EXTREMITY PROSTHESIS}

Electrotactile stimulation might be the most promising way to provide tactile feedback for prostheses, so this section gives a further review of its state-of-the-art studies. In comparison with other tactile feedback techniques mentioned above, electrotactile stimulation has advantages of lower power consumption, light weight and little noise, which makes it suitable to be installed on upper-extremity prostheses. Meanwhile, electric signal acts as the carrier of neural information in the human neural system, so electrotactile stimulation is a direct way to stimulate human PNS by transferring electric signals into the skin and has potential to generate natural tactile perception. Thus, a number of studies about electrotactile feedback has been conducted from different aspects.

\section{A. Role of Tactile Sensation Integration}

Studies to position tactile sensation in the field of prosthetic control mainly focus on functionality improvement and body ownership enhancement. Usually, tactile sensation is expected by prosthesis users in practical use, although there is not a consensus of its effectiveness on performance improvement in academia. Whether integrating tactile sensation would improve the functionality performance of prostheses is still a matter of some controversy. Some reported that the success rate of grasp increased with tactile sensation feedback, while some others pointed out that there was not much difference of the task performances compared with non-feedback control. In order to objectively evaluate the role of tactile sensation in prosthetic control, Jorgovanovic et al. conducted virtual grasping experiments in which the feedback of grasping force was given by electrotactile stimulation and the visual and auditory feedback were eliminated [113]. The outcome confirmed the benefits of tactile sensation in prosthesis force control. In another study, tactile sensation was proposed to build up and update an internal model of feed forward control [114]. Thus, tactile sensation has the potential to improve and facilitate prosthetic control with less effort for the users.

\section{B. Influence Prevention}

Many studies about prosthetic control and tactile stimulation feedback are based on myoelectric prostheses and EEG-based prostheses. As mentioned in section IV-A, myoelectric signals and EEG signals are tiny electric signals, and it is almost bound to be contaminated by electrotactile stimulation, if the signal recording and electrotactile stimulation work on the same body part simultaneously without special processing. Thus, it is necessary to eliminate such influence in practical use.

Various methods were applied to reduce the influence of electrotactile stimulation on myoelectric signals. They can be categorized into two groups: software-based solutions, such as signal processing algorithms; hardware-based solutions, such as blanking/blocking window [115]. Filter-based signal processing algorithms are a common way to restore the performance of myoelectric control, such as the Butterworth filter used in [116], and an adaptive filter based on least mean square (LMS) used in [117]. With regard to hardware-based solutions, time windows were applied between the recording period and stimulating period to avoid overlapping of the myoelectric signal and stimulation signal [118]. Similarly, a method of artificial blanking with three data segmentation approaches was proposed and proved to be an effective way to eliminate the influence of stimulation signal on EMG pattern recognition [119]. Additionally, optimization of stimulation waveform and the electrode design may also help to reduce the interaction of those two electric signals [117].

Apart from above methods, novel prostheses based on nonelectric signal are expected to avoid signal interaction from the very root. For example, sonomyography-based technology has potential to be applied to hand prosthesis [120]. Ultrasound is able to detect changes of muscle thickness in real time, and has no interaction with electric stimulation signals [121]. While, of course, there is usually a long way to go before a novel technology can be applied practically.

\section{Modality Coding}

Natural sensation might be achieved by invasive electrodes [16], while surface electrodes usually generate needlelike, buzz or numb feelings instead. In this case, modality coding is adopted by non-invasive stimulations to provide sensation feedback. Modality coding is to map various stimulation modes on subject's body to represent different tactile sensations.

With regard to electrotactile stimulation, adjustable signal factors include frequency, amplitude, pulse width and wave form. For multi-channel stimulation, the allocation and combination of electrodes can also be taken into consideration. Difference between low frequency $(<30 \mathrm{~Hz})$ and high $(50-100 \mathrm{~Hz})$ frequency can be effectively aware by subjects [122], [123]. Amplitude and wave width are generally used to represent intensity difference such as force feedback. Square wave is commonly used in electrotactile feedback experiments [122], while few comparisons of the impact of different stimulation wave forms can be found in literature so far. Spatial coding is usually adopted by multi-channel system in which the allocation and combination of electrodes are utilized to form different modes and subjects can achieve tactile information by recognizing the stimulation area of the working electrode(s) among all the distributed electrodes [124]. Moreover, mixed coding is applied in tactile sensation restoration and encouraging outcomes were achieved [125]. It should be noticed that most of stimulations are applied for force feedback, only a few of studies concern other tactile sensations, such as texture and shape. Despite the significance of force feedback for grasp tasks, the restoration of other kinds of tactile sensations is also important, because recognizing an object's properties without visual monitoring can enhance a feeling of body ownership and improve the quality of life for people who suffer from hand loss [126]. 


\section{Hybrid Feedback}

Considering the characteristics of different feedback interfaces, attempts have been made to apply more than one type of feedback for sensation delivery. For example, Marco D'Alonzo et al. proposed a hybrid vibro-electrotactile (HyVE) approach which combined vibrotactile stimulator and electrotactile stimulator together to provide sensory feedback, and the experimental outcome was better or comparable to single stimulation [127], [128].

\section{CONCLUDING REMARKS}

Few existing prosthetic hands provide effective tactile sensation feedback to users, which impedes their performance and acceptance. Some tactile feedback techniques and devices have been proposed to address the issue, but with limited success in clinical use. To have a comprehensive understanding of the whole process of tactile sensation restoration for prosthetic hands, this paper presents the physiology of the human sensory system, followed by a review of available tactile sensing and non-invasive stimulation techniques with their working principles, applications, pros and cons. Then, existing studies of electrotactile stimulation is also reviewed in previous section.

Challenges still exist in tactile sensation restoration. For tactile sensing, more effort is expected to promote artificial sensors to be compatible with human skin. Mechanically flexible, stretchable and micro sensors are desired for installation on small or curve surfaces, such as fingertips. Large-scale sensor arrays are also expected for the fabrication of artificial skin. With regard to tactile stimulation feedback, challenges are natural sensation generation and multi-sensation restoration. Among non-invasive tactile stimulations, electrotactile stimulation is believed to be the most promising technique for its small dimension, low noise and friendly interface with electronics. However, the feeling generated by non-invasive stimulation is not as natural as direct neural stimulation, which may increase the user's cognitive burden. Apart from the way of modality coding mentioned in section V-C, the size, shape and types of electrodes and the physiological condition of the stimulated skin also have influence on the performance of tactile sensation restoration, so that further experiments are necessary to explore effective strategies to achieve more natural tactile sensations. Besides, most studies focus on force feedback currently, while studies of tactile feedback can be extended to various tactile features, such as texture, shape and stiffness. Multiple tactile sensations are expected, furthermore, to be fed back simultaneously just like the way that human skin works. Additionally, objective criteria are desired for the assessment of tactile sensation feedback performance, though the expression of sensation or feeling is a subjective concept. Therefore through unified objective criteria, there is scope for various experimental results to be directly compared. Overall, there is still much room for improvement in the field of tactile sensation restoration. An effective and natural restoration of tactile sensation for prosthetic hands will not only advance clinical use in prostheses, but will also benefit the development of virtual reality, MIS, medical training, and so on.

\section{REFERENCES}

[1] U. Wijk and I. Carlsson, "Forearm amputees' views of prosthesis use and sensory feedback," J. Hand Therapy, vol. 28, no. 3, pp. 269-278, 2015.

[2] B. Peerdeman et al., "Myoelectric forearm prostheses: State of the art from a user-centered perspective," J. Rehabil. Res. Develop., vol. 48 , no. 6, pp. 719-738, 2011.

[3] K. Østlie, I. M. Lesjø, R. J. Franklin, B. Garfelt, O. H. Skjeldal, and P. Magnus, "Prosthesis rejection in acquired major upper-limb amputees: A population-based survey," Disab. Rehabil. Assist. Technol. vol. 7, no. 4, pp. 294-303, 2012.

[4] Touch Bionics Products, accessed on Feb. 2017. [Online]. Available: http://www.touchbionics.com/products

[5] How the Michelangelo Works, accessed on Feb. 2017. [Online]. Available: http://www.ottobockus.com/prosthetics/upper-limb-prosthetics/ solution-overview/michelangelo-prosthetic-hand/

[6] Bebionic Hand Features, accessed on Feb. 2017. [Online]. Available: http://bebionic.com/the_hand/features

[7] H. Liu, "Exploring human hand capabilities into embedded multifingered object manipulation," IEEE Trans. Ind. Informat., vol. 7, no. 3, pp. 389-398, Aug. 2011.

[8] The Touch Sensing Hand Prosthesis of the Next Generation: Vincentevolution 2, accessed on Feb. 2017. [Online]. Available: https://vincentsystems.de/en/prosthetics/vincent-evolution-2/

[9] Robotics and Mechatronics Center: Data Sheet of DLR HAND II accessed on Feb. 2017. [Online]. Available: http://www.dlr.de/rmc/rm/ en/desktopdefault.aspx/tabid-3802/6102_read-8922/

[10] P. J. Kyberd, C. Light, P. H. Chappell, J. M. Nightingale, D. Whatley, and M. Evans, "The design of anthropomorphic prosthetic hands: A study of the southampton hand," Robotica, vol. 19, no. 06, pp. 593-600, 2001.

[11] P. J. Kyberd et al., "Case studies to demonstrate the range of applications of the southampton hand assessment procedure," Brit. J. Occupat. Therapy, vol. 72, no. 5, pp. 212-218, 2009.

[12] LUKE arm: Advanced Prosthetic arm System, accessed on Feb. 2017. [Online]. Available: http://www.mobiusbionics.com/the-luke-arm.html

[13] Cyberhand: System Overview, accessed on Feb. 2017. [Online]. Available: http://www-arts.sssup.it/Cyberhand/introduction/biomechand .htm

[14] Shadow Hand: Key Features, accessed on Feb. 2017. [Online]. Available: https://www.shadowrobot.com/products/dexterous-hand/

[15] D. W. Tan, M. A. Schiefer, M. W. Keith, J. R. Anderson, J. Tyler, and D. J. Tyler, "A neural interface provides long-term stable natural touch perception," Sci. Transl. Med., vol. 6, no. 257, p. 257ra138, 2014.

[16] D. J. Tyler, "Restoring the human touch: Prosthetics imbued with haptics give their wearers fine motor control and a sense of connection," IEEE Spectr., vol. 53, no. 5, pp. 28-33, May 2016.

[17] D. Silvera-Tawil, D. Rye, and M. Velonaki, "Artificial skin and tactile sensing for socially interactive robots: A review," Robot. Auto. Syst., vol. 63, pp. 230-243, Jan. 2015.

[18] P. S. Girão, P. M. P. Ramos, O. Postolache, and J. M. D. Pereira "Tactile sensors for robotic applications," Measurement, vol. 46, no. 3, pp. 1257-1271, 2013.

[19] H. J. Witteveen, H. S. Rietman, and P. H. Veltink, "Vibrotactile grasping force and hand aperture feedback for myoelectric forearm prosthesis users," Prosthetics Orthotics Int., vol. 39, no. 3, pp. 204-212, 2014.

[20] C. Dietrich et al., "Sensory feedback prosthesis reduces phantom limb pain: Proof of a principle," Neurosci. Lett., vol. 507, no. 2, pp. 97-100, 2012.

[21] M. H. Lee and H. R. Nicholls, "Review article tactile sensing for mechatronics-A state of the art survey," Mechatronics, vol. 9, no. 1, pp. 1-31, 1999.

[22] B. T. Nghiem et al., "Providing a sense of touch to prosthetic hands," Plastic Reconstruc. Surgery, vol. 135, no. 6, pp. 1652-1663, 2015.

[23] M. Gasson, B. Hutt, I. Goodhew, P. Kyberd, and K. Warwick, "Invasive neural prosthesis for neural signal detection and nerve stimulation," Int. J. Adapt. Control Signal Process., vol. 19, no. 5, pp. 365-375, 2005.

[24] H. Yousef, M. Boukallel, and K. Althoefer, "Tactile sensing in dexterous robot hands-Review," Sens. Actuators A, Phys., vol. 167, no. 2, pp. 171-187, 2011.

[25] C. Lucarotti, C. M. Oddo, N. Vitiello, and M. C. Carrozza, "Synthetic and bio-artificial tactile sensing: A review," Sensors, vol. 13, no. 2, pp. 1435-1466, 2013. 
[26] M. I. Tiwana, S. J. Redmond, and N. H. Lovell, "A review of tactile sensing technologies with applications in biomedical engineering," Sens. Actuators A, Phys., vol. 179, pp. 17-31, Jun. 2012.

[27] C. Antfolk, M. D’Alonzo, B. Rosén, G. Lundborg, F. Sebelius, and C. Cipriani, "Sensory feedback in upper limb prosthetics," Expert Rev. Med. Devices, vol. 10, no. 1, pp. 45-54, 2013.

[28] J. S. Schofield, K. R. Evans, J. P. Carey, and J. S. Hebert, "Applications of sensory feedback in motorized upper extremity prosthesis: A review," Expert Rev. Med. Devices, vol. 11, no. 5, pp. 499-511, 2014.

[29] S. Aoyagi, T. Tanaka, and M. Minami, "Recognition of contact state of four layers arrayed type tactile sensor by using neural network," in Proc. IEEE Int. Conf. Inf. Acquisition, Aug. 2006, pp. 393-397.

[30] J. Dargahi and S. Najarian, "Human tactile perception as a standard for artificial tactile sensing-a review," Int. J. Med. Robot. Comput. Assist. Surgery, vol. 1, no. 1, pp. 23-35, 2004.

[31] R. S. Dahiya, G. Metta, M. Valle, and G. Sandini, "Tactile sensingFrom humans to humanoids," IEEE Trans. Robot., vol. 26, no. 1, pp. 1-20, Feb. 2010.

[32] L. D. Harmon, "Touch-sensing technology: A review," Soc. Manuf. Eng., pp. 37-57, 1980.

[33] M. H. Lee, "Tactile sensing: New directions, new challenges," Int. J. Robot. Res., vol. 19, no. 7, pp. 636-643, 2000.

[34] L. Osborn, W. W. Lee, R. Kaliki, and N. Thakor, "Tactile feedback in upper limb prosthetic devices using flexible textile force sensors," in Proc. 5th IEEE RAS/EMBS Int. Conf. Biomed. Robot. Biomechatronics, Oct. 2014, pp. 114-119.

[35] A. Cranny, D. Cotton, P. Chappell, S. Beeby, and N. M. White, "Thickfilm force and slip sensors for a prosthetic hand," Sens. Actuators A, Phys., vol. 123, pp. 162-171, Sep. 2005.

[36] P. Wang et al., "A virtual reality surgery simulation of cutting and retraction in neurosurgery with force-feedback," Comput. Methods programs Biomed., vol. 84, no. 1, pp. 11-18, 2006.

[37] M. I. C. Dede, Ö. Selvi, T. Bilgincan, and Y. Kant, "Design of a haptic device for teleoperation and virtual reality systems," in Proc. IEEE Int. Conf. Syst., Man, (SMC), Oct. 2009, pp. 3623-3628.

[38] A. Gupta and M. K. O'Malley, "Design of a haptic arm exoskeleton for training and rehabilitation," IEEE/ASME Trans. Mechatronics, vol. 11, no. 3, pp. 280-289, Jun. 2006.

[39] S. Payandeh and T. Li, "Toward new designs of haptic devices for minimally invasive surgery," in Proc. Int. Congr., vol. 1256, Jun. 2003 , pp. 775-781.

[40] F. J. Rubio-Sierra, R. W. Stark, S. Thalhammer, and W. M. Heckl, "Force-feedback joystick as a low-cost haptic interface for an atomicforce-microscopy nanomanipulator," Appl. Phys. A, vol. 76, no. 6, pp. 903-906, 2003.

[41] M. Jobin et al., "Versatile force-feedback manipulator for nanotechnology applications," Rev. Sci. Instrum., vol. 76, no. 5, p. 053701, 2005.

[42] T. R. Coles, D. Meglan, and N. John, "The role of haptics in medical training simulators: A survey of the state of the art," IEEE Trans. Haptics, vol. 4, no. 1, pp. 51-66, Jan./Feb. 2011.

[43] H.-K. Kim, S. Lee, and K.-S. Yun, "Capacitive tactile sensor array for touch screen application," Sens. Actuators A, Phys., vol. 165, no. 1, pp. 2-7, Jan. 2011

[44] J. M. Romano, K. Hsiao, G. Niemeyer, S. Chitta, and K. J. Kuchenbecker, "Human-inspired robotic grasp control with tactile sensing," IEEE Trans. Robot., vol. 27, no. 6, pp. 1067-1079, Dec. 2011

[45] J. Ulmen and M. Cutkosky, "A robust, low-cost and low-noise artificial skin for human-friendly robots," in Proc. ICRA, May 2010, pp. $4836-4841$

[46] S. Najarian, J. Dargahi, and A. Mehrizi, Artificial Tactile Sensing in Biomedical Engineering. New York, NY, USA: McGraw-Hill, 2009.

[47] J. Engel, J. Chen, and C. Liu, "Development of polyimide flexible tactile sensor skin," J. Micromech. Microeng., vol. 13, no. 3, p. 359, 2003

[48] J. G. D. Silva, A. A. D. Carvalho, and D. D. D. Silva, "A strain gauge tactile sensor for finger-mounted applications," IEEE Trans. Instrum. Meas., vol. 51, no. 1, pp. 18-22, Feb. 2002.

[49] C. Pang et al., "A flexible and highly sensitive strain-gauge sensor using reversible interlocking of nanofibres," Nature Mater., vol. 11, no. 9, pp. 795-801, 2012.

[50] E.-S. Hwang, J.-H. Seo, and Y.-J. Kim, "A polymer-based flexible tactile sensor for both normal and shear load detections and its application for robotics," J. Microelectromech. Syst., vol. 16, no. 3, pp. 556-563, Jun. 2007.
[51] S. Stassi, V. Cauda, G. Canavese, and C. F. Pirri, "Flexible tactile sensing based on piezoresistive composites: A review," Sensors, vol. 14, no. 3, pp. 5296-5332, 2014.

[52] A. Wisitsoraat, V. Patthanasetakul, T. Lomas, and A. Tuantranont, "Low cost thin film based piezoresistive MEMS tactile sensor," Sens. Actuators A, Phys., vol. 139, nos. 1-2, pp. 17-22, 2007.

[53] C.-S. Park, J. Park, and D.-W. Lee, "A piezoresistive tactile sensor based on carbon fibers and polymer substrates," Microelectron. Eng., vol. 86, no. 4, pp. 1250-1253, 2009.

[54] M. Ahmed, M. M. Chitteboyina, D. P. Butler, and Z. Celik-Butler, "MEMS force sensor in a flexible substrate using nichrome piezoresistors," IEEE Sensors J., vol. 13, no. 10, pp. 4081-4089, Oct. 2013.

[55] G. Orengo, L. Giovannini, G. Latessa, G. Saggio, and F. Giannini, "Characterization of piezoresistive sensors for goniometric glove in hand prostheses," in Proc. 1st Int. Conf. Wireless Commun., May 2009, pp. 684-687.

[56] B. J. Kane, M. R. Cutkosky, and G. T. A. Kovacs, "A traction stress sensor array for use in high-resolution robotic tactile imaging," J. Microelectromech. Syst., vol. 9, no. 4, pp. 425-434, Dec. 2000.

[57] K. Noda, K. Hoshino, K. Matsumoto, and I. Shimoyama, "A shear stress sensor for tactile sensing with the piezoresistive cantilever standing in elastic material," Sens. Actuators A, Phys., vol. 127, no. 2, pp. 295-301, 2006.

[58] T. Mei, W. J. Li, Y. Ge, Y. Chen, L. Ni, and M. H. Chan, "An integrated MEMS three-dimensional tactile sensor with large force range," Sens. Actuators A, Phys., vol. 80, no. 2, pp. 155-162, 2000

[59] M. Kalantari, M. Ramezanifard, R. Ahmadi, J. Dargahi, and J. Kövecses, "A piezoresistive tactile sensor for tissue characterization during catheter-based cardiac surgery," Int. J. Med. Robot. Comput. Assist. Surgery, vol. 7, no. 4, pp. 431-440, 2011.

[60] R. Kõiva, M. Zenker, C. Schürmann, R. Haschke, and H. J. Ritter, "A highly sensitive 3D-shaped tactile sensor," in Proc. IEEE/ASME Int. Conf. Adv. Intell. Mechatron., Jul. 2013, pp. 1084-1089.

[61] A. Schmitz, M. Maggiali, M. Randazzo, L. Natale, and G. Metta, "A prototype fingertip with high spatial resolution pressure sensing for the robot iCub," in Proc. 8th IEEE-RAS Int. Conf. Humanoid Robots, Dec. 2008, pp. 423-428.

[62] E. Pritchard, M. Mahfouz, B. Evans, S. Eliza, and M. Haider, "Flexible capacitive sensors for high resolution pressure measurement," in Proc. IEEE Sensors, Oct. 2008, pp. 1484-1487.

[63] H. Muhammad et al., "A capacitive tactile sensor array for surface texture discrimination," Microelectron. Eng., vol. 88, no. 8, pp. 1811-1813, 2011.

[64] A. Nafari, F. A. Ghavanini, M. Bring, K. Svensson, and P. Enoksson, "Calibration methods of force sensors in the micro-Newton range," J. Micromech. Microeng., vol. 17, no. 10, p. 2102, 2007.

[65] J. G. V. da Rocha, P. F. A. da Rocha, and S. Lanceros-Mendez, "Capacitive sensor for three-axis force measurements and its readout electronics," IEEE Trans. Instrum. Meas., vol. 58, no. 8, pp. 2830-2836, Aug. 2009.

[66] M. I. Tiwanaa, A. Shashankb, S. J. Redmondb, and N. H. Lovell, "Characterization of a capacitive tactile shear sensor for application in robotic and upper limb prostheses," Sens. Actuators A, Phys., vol. 165, no. 2, pp. 164-172, 2011.

[67] Y. Wang, K. Xi, G. Liang, M. Mei, and Z. Chen, "A flexible capacitive tactile sensor array for prosthetic hand real-time contact force measurement," in Proc. IEEE Int. Conf. Inf. Autom. (ICIA), Jul. 2014, pp. 937-942.

[68] P. Ueberschlag, "PVDF piezoelectric polymer," Sensor Rev., vol. 21, no. 2, pp. 118-126, 2001.

[69] L. Seminara, M. Capurro, P. Cirillo, G. Cannata, and M. Valle, "Electromechanical characterization of piezoelectric PVDF polymer films for tactile sensors in robotics applications," Sens. Actuators A Phys., vol. 169, no. 1, pp. 49-58, 2011.

[70] J. Dargahi, "A piezoelectric tactile sensor with three sensing elements for robotic, endoscopic and prosthetic applications," Sens. Actuators A Phys., vol. 80, no. 1, pp. 23-30, 2000.

[71] S. B. Lang and S. Muensit, "Review of some lesser-known applications of piezoelectric and pyroelectric polymers," Appl. Phys. A, vol. 85, no. 2 , pp. $125-134,2006$.

[72] C. Li, P.-M. Wu, S. Lee, A. Gorton, M. J. Schulz, and C. H. Ahn, "Flexible dome and bump shape piezoelectric tactile sensors using PVDF-TrFE copolymer," J. Micromech. Syst., vol. 17, no. 2, pp. 334-341, Apr. 2008.

[73] A. Marino, G. G. Genchi, V. Mattoli, and G. Ciofani, "Piezoelectric nanotransducers: The future of neural stimulation," Nano Today, Dec. 2016. 
[74] I. Dakua and N. Afzulpurkar, "Piezoelectric energy generation and harvesting at the nano-scale: Materials and devices," Nanomater. Nanotechnol., vol. 3, p. 21, Aug. 2013.

[75] K. H. Lee et al., "Zinc oxide nanowire-based pressure and temperature sensor," in Proc. 15th IEEE Int. Conf. Nanotechnol., Rome, Italy, Jul. 2015, pp. 901-904.

[76] D. P. J. Cotton, P. H. Chappell, A. Cranny, N. M. White, and S. P. Beeby, "A novel thick-film piezoelectric slip sensor for a prosthetic hand," IEEE Sensors J., vol. 7, no. 5, pp. 752-761, May 2007.

[77] S. Takamuku, G. Gomez, K. Hosoda, and R. Pfeifer, "Haptic discrimination of material properties by a robotic hand," in Proc. IEEE 6th Int. Conf. Develop. Learn., Jul. 2007, pp. 1-6.

[78] S. Omata and Y. Terunuma, "New tactile sensor like the human hand and its applications," Sens. Actuators A, Phys., vol. 35, no. 1, pp. 9-15, 1992.

[79] H. Maekawa, K. Tanie, and K. Komoriya, "A finger-shaped tactile sensor using an optical waveguide," in Proc. Int. Conf. Syst., Man Syst. Eng. Service Humans, Oct. 1993, pp. 403-408.

[80] P. Puangmali, K. Althoefer, L. D. Seneviratne, D. Murphy, and P. Dasgupta, "State-of-the-art in force and tactile sensing for minimally invasive surgery," IEEE Sensors J., vol. 8, no. 4, pp. 371-381, Apr. 2008.

[81] J.-S. Heo, J.-Y. Kim, and J.-J. Lee, "Tactile sensors using the distributed optical fiber sensors," in Proc. 3rd Int. Conf. Sens. Technol., Dec. 2008, pp. 486-490.

[82] Y. Yamada, T. Morizono, Y. Umetani, and H. Takahashi, "Highly soft viscoelastic robot skin with a contact object-location-sensing capability," IEEE Trans. Ind. Electron., vol. 52, no. 4, pp. 960-968, Aug. 2005.

[83] A. Ataollahi, P. Polygerinos, P. Puangmali, L. D. Seneviratne, and K. Althoefer, "Tactile sensor array using prismatic-tip optical fibers for dexterous robotic hands," in Proc. IEEE/RSJ Int. Conf. Intell. Robot. Syst. (IROS), Oct. 2010, pp. 910-915.

[84] M. Ohka, Y. Mitsuya, S. Takeuchi, H. Ishihara, and O. Kamekawa, "A three-axis optical tactile sensor (FEM contact analyses and sensing experiments using a large-sized tactile sensor)," in Proc. IEEE Int. Conf. Robot. Autom., vol. 1, May 1995, pp. 817-824.

[85] A. Massaro, F. Spano, A. Lay-Ekuakille, P. Cazzato, R. Cingolani, and A. Athanassiou, "Design and characterization of a nanocomposite pressure sensor implemented in a tactile robotic system," IEEE Trans. Instrum. Meas., vol. 60, no. 8, pp. 2967-2975, Aug. 2011.

[86] L. Ascari, P. Corradi, L. Beccai, and C. Laschi, "A miniaturized and flexible optoelectronic sensing system for tactile skin," J. Micromech. Microeng., vol. 17, no. 11, p. 2288, 2007.

[87] J. Rossiter and T. Mukai, "A novel tactile sensor using a matrix of LEDs operating in both photoemitter and photodetector modes," in Proc. IEEE Sensors, Nov. 2005, pp. 994-997.

[88] Y. Ohmura, Y. Kuniyoshi, and A. Nagakubo, "Conformable and scalable tactile sensor skin for curved surfaces," in Proc. IEEE Int. Conf. Robot. Autom. (ICRA), May 2006, pp. 1348-1353.

[89] H. N. Sani and S. G. Meek, "Characterizing the performance of an optical slip sensor for grip control in a prosthesis," in Proc. IEEE/RSJ Int. Conf. Intell. Robot. Syst., Sep. 2011, pp. 1927-1932.

[90] J. Kim et al., "Stretchable silicon nanoribbon electronics for skin prosthesis," Nature Commun., vol. 5, Dec. 2014, Art. no. 5747.

[91] B. C.-K. Tee et al., "A skin-inspired organic digital mechanoreceptor," Science, vol. 350, no. 6258, pp. 313-316, 2015.

[92] F. Quandt and F. C. Hummel, "The influence of functional electrical stimulation on hand motor recovery in stroke patients: A review," Experim. Transl. Stroke Med., vol. 6, p. 9, Aug. 2014.

[93] S. Bhattacharyya, M. Clerc, and M. Hayashibe, "A study on the effect of electrical stimulation during motor imagery learning in braincomputer interfacing," in Proc. IEEE Int. Conf. Syst., Man Cybern. (SMC), Budapest, Hungary, Oct. 2016, pp. 002840-002845.

[94] M. Mulvey, H. Fawkner, H. Radford, and M. Johnson, "The use of transcutaneous electrical nerve stimulation (TENS) to aid perceptual embodiment of prosthetic limbs," Med. Hypotheses, vol. 72, no. 2, pp. 140-142, 2009.

[95] M. R. Mulvey, A.-M. Bagnall, P. R. Marchant, and M. I. Johnson, "Transcutaneous electrical nerve stimulation (TENS) for phantom pain and stump pain following amputation in adults: An extended analysis of excluded studies from a Cochrane systematic review," Phys. Ther. Rev., vol. 19, no. 4, pp. 234-244, 2014.
[96] M. R. Mulvey, H. J. Fawkner, H. E. Radford, and M. I. Johnson, "Perceptual embodiment of prosthetic limbs by transcutaneous electrical nerve stimulation," Neuromodulat., Technol. Neural Interface, vol. 15 , no. 1, pp. 42-47, 2012.

[97] M. Mulvey, H. Fawkner, and M. I. Johnson, "An investigation into the perceptual embodiment of an artificial hand using transcutaneous electrical nerve stimulation (TENS) in intact-limbed individuals," Technol. Health Care, vol. 22, no. 2, pp. 157-166, 2014.

[98] G. Chai, X. Sui, S. Li, L. He, and N. Lan, "Characterization of evoked tactile sensation in forearm amputees with transcutaneous electrical nerve stimulation," J. Neural Eng., vol. 12, no. 6, p. 066002, 2015.

[99] H. Xu, D. Zhang, J. C. Huegel, W. Xu, and X. Zhu, "Effects of different tactile feedback on myoelectric closed-loop control for grasping based on electrotactile stimulation," IEEE Trans. Neural Syst. Rehabil. Eng., vol. 24, no. 8, pp. 827-836, Aug. 2016.

[100] S. Bhattacharyya, M. Clerc, and M. Hayashibe, "A study on the effect of electrical stimulation as a user stimuli for motor imagery classification in brain-machine interface," Eur. J. Transl. Myol., vol. 26, no. 2, pp. 165-168, 2016.

[101] S. G. Meek, S. C. Jacobsen, and P. P. Goulding, "Extended physiologic taction: Design and evaluation of a proportional force feedback system," J. Rehabil. Res. Develop., vol. 26, no. 3, pp. 53-62, 1989.

[102] J. E. Conzelman, Jr., H. B. Ellis, and C. W. Obrien, "Prosthetic device sensory attachment," U.S. Patent 2656545 , Oct. 27, 1953.

[103] E. Rombokas, C. E. Stepp, C. Chang, M. Malhotra, and Y. Matsuoka, "Vibrotactile sensory substitution for electromyographic control of object manipulation," IEEE Trans. Biomed. Eng., vol. 60, no. 8, pp. 2226-2232, Aug. 2013.

[104] A. Chatterjee, V. Aggarwal, A. Ramos, S. Acharya, and N. V. Thakor, "A brain-computer interface with vibrotactile biofeedback for haptic information," J. Neuroeng. Rehabil., vol. 4, no. 1, p. 40, 2007.

[105] F. Cincotti et al., "Vibrotactile feedback for brain-computer interface operation," Comput. Intell. Neurosci., vol. 2007, p. 7, Jan. 2007.

[106] S. Schätzle and B. Weber, "Towards vibrotactile direction and distance information for virtual reality and workstations for blind people," in Proc. Int. Conf. Univ. Access Human-Comput. Interact., 2015, pp. $148-160$

[107] A. Chang, S. O'Modhrain, R. Jacob, E. Gunther, and H. Ishii, "Comtouch: Design of a vibrotactile communication device," in Proc. 4th Conf. Designing Interact. Syst. Processes, Pract. Methods, Techn., 2002, pp. 312-320.

[108] C. Cipriani, F. Zaccone, S. Micera, and M. C. Carrozza, "On the shared control of an EMG-controlled prosthetic hand: Analysis of user-prosthesis interaction," IEEE Trans. Robot., vol. 24, no. 1, pp. 170-184, Feb. 2008.

[109] S. Casini, M. Morvidoni, M. Bianchi, M. Catalano, G. Grioli, and A. Bicchi, "Design and realization of the CUFF-Clenching upperlimb force feedback wearable device for distributed mechano-tactile stimulation of normal and tangential skin forces," in Proc. IEEE/RSJ Int. Conf. Intell. Robot. Syst. (IROS), Oct. 2015, pp. 1186-1193.

[110] R. L. J. Hollis, "Magnetic levitation haptic interface system," U.S. Patent 8497767, Jul. 30, 2013.

[111] F. Arafsha, L. Zhang, H. Dong, and A. El Saddik, "Contactless haptic feedback: State of the art," in Proc. IEEE Int. Symp. Haptic, Audio Vis. Environ. Games (HAVE), Oct. 2015, pp. 1-6.

[112] P. Kutilek, J. Hybl, J. Kauler, and S. Viteckova, "Prosthetic 6-DOF arm controlled by EMG signals and multi-sensor system," in Proc. 15th Int. Symp. MECHATRONIKA, Dec. 2012, pp. 1-5.

[113] N. Jorgovanovic, S. Dosen, D. J. Djozic, G. Krajoski, and D. Farina, "Virtual grasping: Closed-loop force control using electrotactile feedback," Comput. Math. Methods Med., vol. 2014, Jan. 2014, Art. no. 120357

[114] S. Dosen et al., "Building an internal model of a myoelectric prosthesis via closed-loop control for consistent and routine grasping," Experim. Brain Res., vol. 233, no. 6, pp. 1855-1865, 2015.

[115] F. Widjaja, C. Y. Shee, P. Poignet, and W. T. Ang, "FES artifact suppression for real-time tremor compensation," in Proc. IEEE Int. Conf. Rehabil. Robot., Jun. 2009, pp. 53-58.

[116] M. Peruzzini, M. Germani, and M. Mengoni, "Electro-tactile device for texture simulation," in Proc. IEEE/ASME Int. Conf. Mechatron. Embedded Syst. Appl. (MESA), Jul. 2012, pp. 178-183.

[117] L. Jiang, Q. Huang, J. Zhao, D. Yang, S. Fan, and H. Liu, "Noise cancellation for electrotactile sensory feedback of myoelectric forearm prostheses," in Proc. IEEE Int. Conf. Inf. Autom. (ICIA), Jul. 2014, pp. 1066-1071. 
[118] S. Dosen, M.-C. Schaeffer, and D. Farina, "Time-division multiplexing for myoelectric closed-loop control using electrotactile feedback," J. Neuroeng. Rehabil., vol. 11, no. 1, p. 138, Sep. 2014.

[119] C. Hartmann, S. Došen, S. Amsuess, and D. Farina, "Closed-loop control of myoelectric prostheses with electrotactile feedback: Influence of stimulation artifact and blanking," IEEE Trans. Neural Syst. Rehabil. Eng., vol. 23, no. 5, pp. 807-816, May 2015.

[120] N. Akhlaghi et al., "Real-time classification of hand motions using ultrasound imaging of forearm muscles," IEEE Trans. Biomed. Eng., vol. 63, no. 8, pp. 1687-1698, Aug. 2016.

[121] Y. Fang, N. Hettiarachchi, D. Zhou, and H. Liu, "Multi-modal sensing techniques for interfacing hand prostheses: A review," IEEE Sensors J., vol. 15, no. 11, pp. 6065-6076, Nov. 2015

[122] A. H. Arieta, H. Yokoi, T. Arai, and Y. Wenwei, "FES as biofeedback for an EMG controlled prosthetic hand," in Proc. TENCON IEEE Region Conf., Nov. 2005, pp. 1-6.

[123] L. P. Paredes, S. Dosen, F. Rattay, B. Graimann, and D. Farina, "The impact of the stimulation frequency on closed-loop control with electrotactile feedback," J. Neuroeng. Rehabil., vol. 12, no. 1, pp. 1-16, 2015.

[124] M. Isaković, M. Belić, M. Štrbac, and T. Keller, "Electrotactile feedback improves performance and facilitates learning in the routine grasping task," Eur. J. Transl. Myol., vol. 26, no. 3, p. 6069, 2016.

[125] S. Dosen et al., "Multichannel electrotactile feedback with spatial and mixed coding for closed-loop control of grasping force in hand prostheses," IEEE Trans. Neural Syst. Rehabil. Eng., to be published.

[126] S. Raspopovic et al., "Restoring natural sensory feedback real-time bidirectional hand prostheses," Sci. Transl. Med., vol. 6, no. 222, p. 222ra19, 2014.

[127] M. D'Alonzo, S. Dosen, C. Cipriani, and D. Farina, "HyVE: Hybrid vibro-electrotactile stimulation for sensory feedback and substitution in rehabilitation," IEEE Trans. Neural Syst. Rehabil. Eng., vol. 22, no. 2, pp. 290-301, Mar. 2014.

[128] M. D'Alonzo, S. Dosen, C. Cipriani, and D. Farina, "HyVEHybrid vibro-electrotactile stimulation-Is an efficient approach to multi-channel sensory feedback," IEEE Trans. Haptics, vol. 7, no. 2, pp. 181-190, Apr./Jun. 2014.

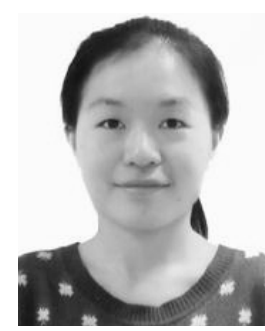

Kairu $\mathbf{L i}$ received the B.S. degree in automation and the M.S. degree in control engineering and control theory from the Dalian University of Technology, China, in 2012 and 2015, respectively. She is currently pursuing the Ph.D. degree, under the supervision of Prof. H. Liu, with the School of Computing from the University of Portsmouth, U.K. Her research interests include wearable sensor systems, electrotactile stimulation, sensory feedback in prosthetics, and rehabilitation.

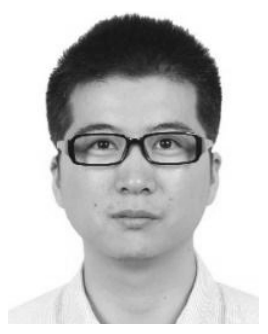

Yinfeng Fang the M.S. degree in pattern recognition and intelligent system from the Zhejiang University of Technology, Hangzhou, China, in 2012, and the Ph.D. degree in computer science from the University of Portsmouth, Portsmouth, U.K. 2015. $\mathrm{He}$ joined the Intelligent System and Biomedical Robotics Group, University of Portsmouth, in 2015, as a Senior Research Associate. His research interest is on bio-signal acquisition and processing, humanmachine interface, and machine learning.

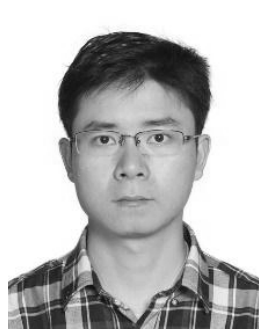

Yu Zhou received the B.S. degree from the College of Mechanical and Electrical Engineering, Central South University, Changsha, China, in 2014. He is currently pursuing the Ph.D. degree with the School of Mechanical Engineering, Shanghai Jiao Tong University, Shanghai, China. His research interests include intelligent system, biological signal processing, and electrical stimulation.

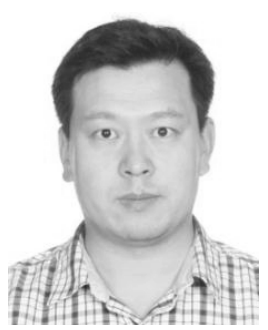

Honghai Liu received the Ph.D. degree in robotics from King's College London, London, U.K., in 2003. He held the position of research appointments with the Universities of London and Aberdeen, and project leader appointments in the large-scale industrial control and system integration industry. He joined the University of Portsmouth, Portsmouth, U.K., in 2005. His current research interests include approximate computation, pattern recognition, intelligent video analytics and robotics, and their practical applications with an emphasis on approaches that could make contributions to the intelligent connection of perception to action using contextual information.

Dr. Liu has authored over 200 peer-reviewed international journal and conference papers and received four best paper awards. He is an Associate Editor of the IEEE TRANSACTIONS ON INDUSTRIAL INFORMATICS, the IEEE TRANSACTIONS ON SySTEMS, MAN AND Cybernetics-PART C, the International Journal of Fuzzy Systems, and the KES Journal. 